\title{
Surface Enhanced Resonance Raman Spectroscopy (SERRS) for Probing Through Plastic and Tissue Barriers Using a Handheld Spectrometer
}

\section{Authors}

Fay Nicolson ${ }^{1}$, Lauren E. Jamieson ${ }^{1}$, Samuel Mabbott ${ }^{1}$, Konstantinos Plakas ${ }^{2}$, Neil C. Shand ${ }^{3}$, Michael R. Detty ${ }^{2}$, Duncan Graham ${ }^{1}$, Karen Faulds ${ }^{1 *}$

1. Department of Pure and Applied Chemistry, Technology and Innovation Centre, University of Strathclyde, 99 George Street, Glasgow G1 1RD, UK.

2. Department of Chemistry, University at Buffalo, The State University of New York, New York 14260, United States

3. Defence Science Technology Laboratory, Porton Down, Salisbury, SP4 0JQ, UK.

Corresponding author:

Karen Faulds, Department of Pure and Applied Chemistry, Technology and Innovation Centre, University of Strathclyde, 99 George Street, Glasgow G1 1RD, UK.

E-mail: karen.faulds@strath.ac.uk

\section{Abstract}

The ability to probe through barriers and tissue non-invasively is an urgent unmet need in both the security and biomedical imaging fields. Surface enhanced Raman spectroscopy (SERS) has been shown to yield superior enhancement in signal over conventional Raman techniques. Furthermore, by utilising a resonant Raman reporter to produce surface enhanced resonance Raman spectroscopy (SERRS), even greater enhancement in chemical signal can be generated. Here we show the benefit of using red-shifted chalcogenpyrylium based Raman reporters for probing through large thicknesses of plastic and tissue barriers using a conventional Raman instrument. Furthermore, the benefit of using a resonant Raman reporter for superior levels of through barrier detection is demonstrated, thus we aim to show the advantage of using resonant nanotags in combination with conventional Raman spectroscopy to probe through plastic and tissue barriers. Raman signals were collected from SERRS active nanotags through plastic thicknesses of up to $20 \mathrm{~mm}$, as well as the detection of the same SERRS nanotags through up to $10 \mathrm{~mm}$ of tissue sections using a handheld conventional Raman spectrometer. The ability to detect SERRS-active nanotags taken up into ex vivo tumour models known as multicellular tumour spheroids (MTS), through depths of $5 \mathrm{~mm}$ of tissue was also shown. The advantages of applying multivariate analysis for through barrier detection when discriminating analytes with similar spectral features as the barrier is also clearly demonstrated. To the best of our knowledge, this is the first report of the assessment of the maximum level of through barrier detection using a conventional handheld Raman instrument for SERS applications as well as demonstration of the power of resonant nanotags for probing through barriers using conventional Raman spectroscopy.

\section{Introduction}


Raman spectroscopy provides sensitive, molecularly specific vibrational information, however it is an inherently weak scattering process. ${ }^{1}$ Through the use of molecularly specific Raman reporters adsorbed on the surface of metallic nanostructures, e.g. gold nanoparticles (AuNPs), surface enhanced Raman scattering (SERS) provides a means of enhancing the Raman scattering process by several orders of magnitude. ${ }^{1}$ Moreover, by utilising a laser that corresponds to an electronic transition of the analyte, further enhancement can be achieved by surface enhanced resonance Raman scattering (SERRS). Not only has SERRS been reported to produce vibrational fingerprint spectra with enhancements up to $10^{14}$, the nanoparticles can also quench the fluorescence that can be an issue with resonant enhancement. ${ }^{2,}{ }^{3}$ Such properties are useful for the purpose of this work where the ability to detect vibrational spectra through barriers is reported.

SERS has been applied in a wide array of biomedical and security applications including the detection of antimicrobial resistant pathogens, ${ }^{3}$ bacterial spores ${ }^{4}$ in vivo imaging ${ }^{5}$ and in the detection of explosives. ${ }^{6}$ From a security perspective, SERS has been useful in the detection of explosives including dinitrotoluene (DNT) ${ }^{7}$ and trinitrotoluene (TNT). ${ }^{6}$ SERS has been used extensively in applications involving biomedical imaging and nanotags functionalised with biomolecules such as antibodies have assisted in the targeted imaging of numerous cancers in vivo including breast, ${ }^{8}$ ovarian ${ }^{9}$ as well as photothermal applications. ${ }^{10}$

However, the ability to perform SERS analysis for either security or biomedical applications not only relies upon the effectiveness of the SERS probes themselves, but also on the efficiency and portability of the Raman instrumentation. ${ }^{11}$ Confocal techniques are frequently applied in order to obtain signal through a barrier, for example plastic or tissue. In this instance, the microscope is focused to a single depth and spectra recorded at each z-plane..$^{12}$ It is also possible to use a defocused beam in which a positive and negative SERS response can be obtained quickly. ${ }^{11}$ However, such mapping experiments are typically preformed using benchtop instruments which are often bulky and lack the portability required to facilitate measurements in the field or clinic. As such, there has been a considerable shift towards advancements in handheld Raman instrumentation in recent years. This is in part due to their ease of use, portability, lower cost and user friendly nature. ${ }^{8,13}$ As a consequence, there has been a substantial increase in the number of portable Raman instruments available on the market. ${ }^{14}$

Spatially offset Raman spectroscopy (SORS) is a relatively new technique that is particularly useful for probing through barriers, specifically plastic ${ }^{15,16}$ and tissue. ${ }^{17}$ SORS has been used for the detection of ammonium nitrate through $4.5 \mathrm{~mm}$ of tissue and in the detection of counterfeit alcohol and also to the transcutaneous, ${ }^{18}$ and in vivo analysis of bone and bone disease. ${ }^{19}$ Using a handheld SORS instrument, we have reported the ability to detect ethanol through up to $21 \mathrm{~mm}$ of plastic. ${ }^{13}$ Surface enhanced spatially offset Raman spectroscopy (SESORS) combines SERS and SORS and is useful in the detection of SERS nanotags through considerable thicknesses of tissue. ${ }^{20,21}$ By combining SERRS with SORS to yield surface enhanced spatially offset resonance Raman spectroscopy (SESORRS), SERRS nanotags present in ex vivo breast cancer tumour models were detected through depths of 15 
$\mathrm{mm}$ of tissue. ${ }^{22,23}$ However, in this instance, we aim to demonstrate the powerful capabilities of handheld conventional Raman instrumentation for detecting SERS analytes at significant depth, without the need for SORS techniques.

We have recently reported the use of a handheld conventional Raman instrument for the detection of ethanol through thicknesses of up to $9 \mathrm{~mm}$ of plastic. ${ }^{13} \mathrm{In}$ combination with multivariate analysis in the form of principal component analysis (PCA), signal was detected through greater depths than could be deconvoluted by eye. Using the same handheld Raman instrument utilised in the work reported here, Van Duyne and co-workers employed SERS for the detection and identification of hair dyes, ${ }^{24}$ and in part, for the detection and quantification of intravenous drug therapies. ${ }^{25}$ Herein we discuss the use of the same handheld Raman instrument, with back-scattering optics, for the detection of SERRS nanotags through plastic and tissue barriers as well as for the detection of SERRS nanotags taken up into ex vivo tumour models. Furthermore, we describe the benefit of using resonant Raman reporters for probing through greater thicknesses. The results presented here are particularly impressive due to the use of a handheld instrument, which unlike microscope-based systems has a fixed focal depth, and in theory, should limit its ability to probe through deeper layers. To the best of our knowledge, this is the first assessment of the use of handheld Raman, rather than SORS, for detecting SERS nanotags through the maximum thickness of plastic and tissue barriers.

\section{Materials and Methods}

\section{Synthesis of SERS nanotags}

All chemicals and small molecule Raman reporters were purchased from Sigma Aldrich unless otherwise stated. AuNPs with an average diameter of $78 \mathrm{~nm}$ were synthesised using a previously reported method. ${ }^{22}$ AuNPs were characterised using extinction spectroscopy and had an LSPR of $552 \mathrm{~nm}$. Chalcogenpyrylium-based dyes were synthesized according to previously reported methods. ${ }^{26}$ Their chemical structures are shown in the supporting information (Figure S1). They are named according to their absorbance maxima. For example, dye823 has an absorbance maxima of $823 \mathrm{~nm}$. Dyes 676, 823 and 959 were prepared by dissolving the solid in anhydrous N,N-dimethylformamide (DMF, 99.8\%) to produce a $1 \mathrm{mM}$ stock. Subsequent dilutions were then carried out using DMF and $\mathrm{dH}_{2} \mathrm{O}$ (50:50). Raman reporters 1,2-bis(4-pyridyl)ethylene (BPE) and 4,4-azopyridine (AZPY) were prepared by dissolving the solid in ethanol to produce a $10 \mathrm{mM}$ stock. Subsequent dilutions were carried out using $\mathrm{dH}_{2} \mathrm{O}$. Dyes were characterized using extinction spectroscopy (Agilent Cary 60) to determine their $\lambda$ max. BPE and AZPY are non-resonant Raman reporters.

Prior to dye addition, NPs were concentrated by centrifugation $(1 \mathrm{~mL}$ aliquots, 5000 RPM, 10 mins) and resuspended in $500 \mu \mathrm{L}$ of water. Investigation of the nanotags for SERS applications was carried out by adding each reporter to the AuNPs. The total final volume of each nanotag sample was $1 \mathrm{~mL}$. A final dye concentration of $300 \mathrm{nM}$ 
was used, keeping the dye concentration as low as possible to exploit the benefit of using a Raman reporter which is in resonance with the laser. The SERS spectra for each of the five Raman reporter molecules is shown in the supporting information, Figure S2.

\section{SERS measurements}

All SERS measurements were carried out using a handheld CBEx spectrometer, 785$\mathrm{nm}$ laser excitation wavelength, from Snowy Range Instruments (now Metrohm). Measurements involving plastic were obtained using a 3-s integration time. Tissue experiments were carried out using a 5-s integration time. A point and shoot adaptor with a single element lens and a numerical aperture of 0.5 was fitted for through barrier detection. This gave an average laser power of $43 \mathrm{~mW}$. The focal spot of the CBEx was measured using a beam profiler (BeamMap 2-XYZ scanning slit system 190$2,500 \mathrm{~nm}$, Data-Ray Inc.). The CBEx instrument used in this instance had a spot size of 50-60 microns at a focal distance of $0.5 \mathrm{~cm}$.

\section{Through barrier detection of SERS nanotags obscured by plastic}

Large transparent polyethylene terephthalate (PET) and blue opaque coloured polypropylene (PP) plastic sheets were purchased from a local art shop and then cut up into smaller rectangular pieces $(10.5 \times 3 \mathrm{~cm}$, thickness $1 \mathrm{~mm})$. The sheets were mounted on a stage and clamped together to create the desired thickness. They were then brought into contact with the laser using the point and shoot adaptor (supporting information, Figure S3). This ensured that there was no air/space between the plastic and instrument. A glass vial containing the nanotags was placed behind the plastic sheets. The glass vials had a $15-\mathrm{mm}$ diameter, 1-mm thickness, and a height of 25 $\mathrm{mm}$ (including lid). To determine the maximum thickness of plastic the instruments could detect the SERS nanotags through, measurements were carried out using varying thicknesses of plastic. The thickness of plastic was increased by $1 \mathrm{~mm}$ for each set of spectral acquisitions until the maximum thickness at which the instrument could detect the SERS signal from the nanotags was determined.

\section{Cell culture}

MCF7 cells (ECACC 86012803) were obtained from the Institute of Genetics and Molecular Medicine at the University of Edinburgh. The cell line was originally purchased from Sigma. MCF7 human breast cancer cells were cultured in Rosewell Park Memorial Institute medium (RPMI) supplemented with $1 \%$ penicillin/streptomycin $(10,000$ units $/ \mathrm{mL}), 1 \%$ fungizone, and $10 \%$ heat-inactivated fetal bovine serum (FBS). Cells were incubated at $37{ }^{\circ} \mathrm{C}$ and $5 \% \mathrm{CO}_{2}$ in a humidified incubator. Cells at a confluence of ca. $90 \%$ growing in a T75 flask were incubated overnight with $13.7 \mathrm{pM}$ of AuNP. The following day, cells were trypsinised and re-suspended in medium to give a concentration of ca. $2.4 \times 10^{6}$ cells cells $/ \mathrm{mL}$. Multicellular tumour spheroids (MTS) were grown using a hanging drop technique by pipetting $20 \mu \mathrm{L}$ drops of this cell suspension onto the lid of a petri dish with ca. $12 \mathrm{~mL}$ of medium added to the dish. The lid was placed on the dish and MTS grew over a period of 7 days at $37{ }^{\circ} \mathrm{C}$ and $5 \% \mathrm{CO}_{2}$ in a humidified incubator. Medium was removed from the drops and replaced after 2 days. 


\section{Through barrier detection of SERS nanotags obscured by tissue}

Pork loin tissue was obtained from a local butcher and cut into sections (roughly 3.5 $\mathrm{cm}$ inches $\times 4 \mathrm{~cm}$ with varying thicknesses). Tissue experiments were performed using two approaches. For measurements involving a cuvette, $350 \mu \mathrm{L}$ of each NP-Dye solution was pipetted into a Suprasil quartz micro cuvette, path length $1 \mathrm{~mm}$, chamber volume $350 \mu \mathrm{L}$. Tissue samples of varying thicknesses were then placed in front of the cuvette. The point and shoot adaptor was brought into contact with the tissue samples, thus ensuring there was no space between the instrument and the tissue. The experimental set up is described in the supporting information, Figure S4.

For mapping experiments, MTS models containing the SERRS nanotags were placed directly onto a section of tissue and left to equilibrate for 10 mins. Following this, $5 \mathrm{~mm}$ of tissue was then placed on top of the tissue layer containing the MTS models. The two-layer sample was then brought into contact with the laser via the point and shoot adaptor, supporting information, Figure S5. The handheld CBEx instrument was positioned above the tissue samples with the laser pointing down onto the tissue (Figure S3). This set up is more representative of an in vivo approach compared to that using the cuvette. An $x-y$-positioning stage was used to enable Raman mapping of either the SERRS active nanotags taken up into MTS through $5 \mathrm{~mm}$ of tissue. The stage was moved in $1 \mathrm{~mm}$ steps create a $10 \times 10$ pixel image (total area $1 \mathrm{~cm}^{2}$ ).

\section{Data processing}

All spectra were processed using Matlab software (version 2017a, The MathWorks, Natrick, MA, USA). Principal component analysis (PCA) was applied to data involving plastic barriers. Fifteen spectra (five replicates, three samples) of plastic at a given thickness were obtained followed by 15 spectra (five replicates, three samples) of SERS nanotags obscured by plastic of the same thickness. Preprocessing involved truncating and scaling the spectra, before applying the first-order derivative coupled with Savitzky-Golay smoothing. The first-order derivative was used in PCA to remove slight variances in the background, which were found to affect the resulting zero- order PCA plots. ${ }^{27}$ For mapping experiments, spectra were truncated, baselined and smoothed using Savitzky-Golay filtering before the intensity at $1596 \mathrm{~cm}^{-1}$ at each of the $1 \mathrm{~mm}$ steps was plotted as a combination surface/contour false colour $2 \mathrm{D}$ heat map.

\section{Results and discussion}

We have previously reported the use of red-shifted chalcogenpyrylium based Raman reporters for both SERS ${ }^{1,28,26}$ and surface enhanced spatially offset Raman spectroscopy (SESORS) applications using NIR Raman excitation. ${ }^{22}$ By controlling the length of the number of $\mathrm{sp}^{2}$ carbons in the aliphatic backbone and the choice of chalcogen atoms in the ring system, it is possible to tune the absorption maximum of the Raman reporter into the near infrared (NIR). More specifically the Raman reporter can be synthesised to be in resonance with the laser wavelength of the Raman instrument, thus making them particularly attractive for SERRS applications. Furthermore, these chalgoenpyrylium dyes have also been shown to outperform commercially available non-resonant Raman reporters including BPE (1,2-bis(4- 
pyridyl)ethylene) and AZPY (4,4-azopyridine). ${ }^{26,22}$ These studies focused on the attractiveness of these red-shifted chalcogenpyrylium based Raman reporters for SERS applications in the NIR region, however the results presented here focus on the use of these nanotags for probing through significant depths using conventional handheld Raman. In recent years there has been a shift towards the use of SORS for probing through barriers ${ }^{29,30}$, however this work explores the use of conventional Raman, rather than SORS, to detect SERS signals at depth through plastic and tissue.

To demonstrate the benefit of using a resonant Raman reporter to probe through large thicknesses of both plastic and tissue, nanotag solutions were prepared by functionalising AuNPs with each of the five Raman reporter molecules, i.e. dye 676 nanotag solution contained AuNPs functionalised with dye 676. The dyes are named according to their absorbance maximum, i.e. dye 676 has a $\lambda_{\max }$ at $676 \mathrm{~nm}$. Each nanotag solution was initially obscured by plastic barriers and the maximum thickness through which the nanotags could be detected using a handheld Raman instrument, both by eye and using chemometrics, was determined. Figure 1 shows the tracking of dye 823 nanotags through thicknesses of up to $21 \mathrm{~mm}$ of PET (a) and up to $10 \mathrm{~mm}$ of blue PP (b). In both instances the spectrum at the top is the plastic reference spectrum of the barrier and the spectrum at the bottom is of the dye823 nanotags. The handheld instrument used in this work has a laser excitation wavelength of $785 \mathrm{~nm}$, thus nanotags containing dye 823 have an electronic transition close to that of the laser line and are therefore in resonance with the handheld instrument and were expected to generate the largest level of though barrier detection. From previous reports, ${ }^{22,26}$ it was also expected that the off-resonant chalcogenpyrylium-based reporters would also provide superior levels of through barrier detection compared to BPE and AZPY. The PP spectrum is very weak and has a poor signal to noise ratio, with little meaningful spectral characteristics. It is believed that this is due to optical limitations of the handheld instrument, which prevents it from resolving the peaks effectively. However, in this instance, the main aim was not to detect PP peaks, but to use it as a barrier to block SERS signal from the nanotags. As expected, when the thickness of either PET or PP increases, the spectral contribution of dye 823 to the acquired spectrum diminishes. Through thicknesses of $5 \mathrm{~mm}$ PET, dye 823 is clearly observed in the acquired spectrum $\left(1179\right.$ and $\left.1596 \mathrm{~cm}^{-1}\right)$. Similar results are observed when dye 823 is tracked through $4 \mathrm{~mm}$ of PP. However, as the thickness of plastic is increased, it become difficult to visually detect dye 823 . This is particularly true for through barrier detection involving PET, since, although a shoulder that corresponds to that of the dye can be seen in the 1590 to $1600 \mathrm{~cm}^{-1}$ region through thicknesses of $10 \mathrm{~mm}$ of PET, the dye peak at $1596 \mathrm{~cm}^{-1}$ is in close proximity to the plastic peak at $1614 \mathrm{~cm}^{-1}$. 
a)

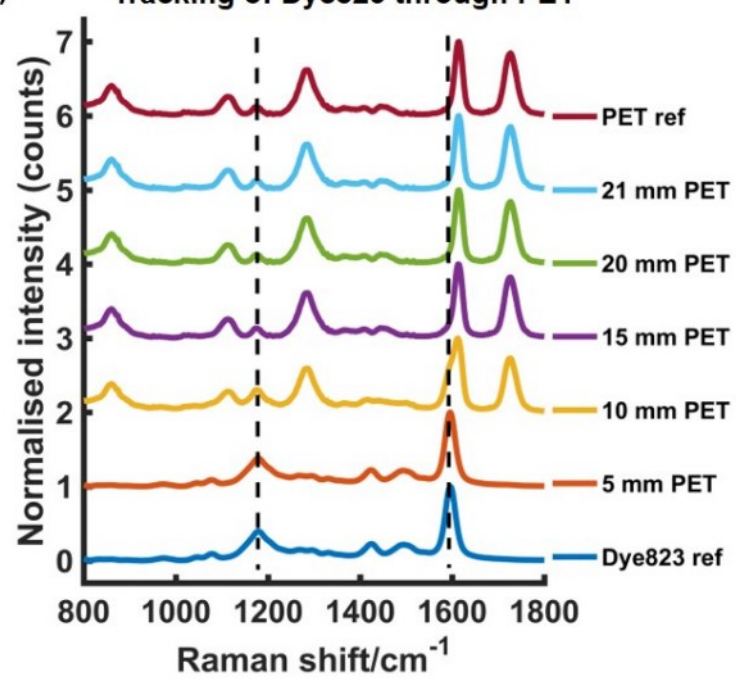

b) Tracking of Dye823 through PP

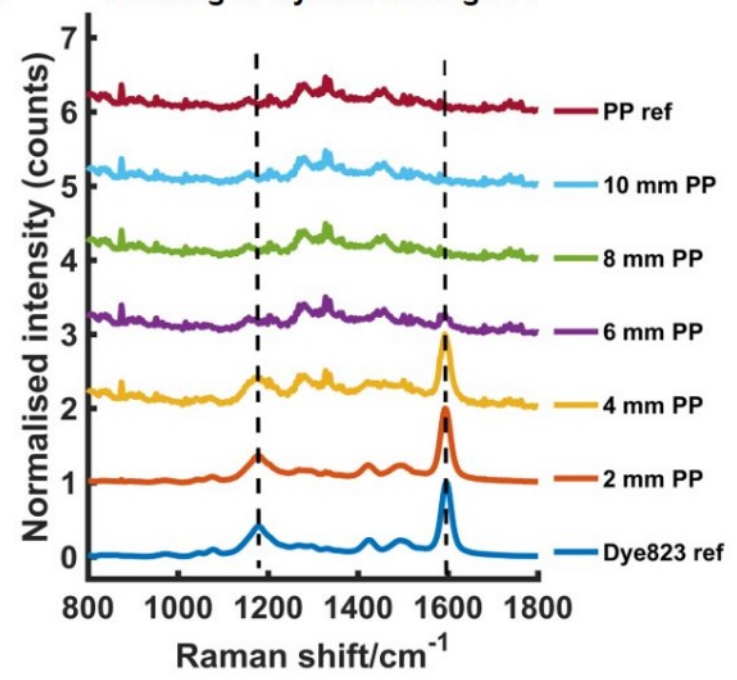

Figure 1 - The tracking of dye 823 through PET (a) and PP (b). In both instances, the spectra at the bottom refers dye 823 nanotags and the spectra at the top refers to PET and PP respectively. The dashed line refers to the characteristic dye peaks at 1179 and $1596 \mathrm{~cm}^{-1}$. Spectra were averaged, normalised to the maximum peak in the spectrum and stacked for clarity. Both sets of stacked spectra show the varying contribution of dye 823 and plastic to the acquired spectra as the thickness of the barrier is increased. Measurements were performed using 3 samples, 5 replicates, at a laser excitation wavelength of $785 \mathrm{~nm}$, average laser power $43 \mathrm{~mW}$, and 3-s integration time, five accumulations.

To determine the maximum depth at which dye 823 could be detected, multivariate analysis in the form of PCA was applied. PCA is a well-known chemometric approach that is used to reduce the dimensionality of multivariate data whilst preserving most of the variance, i.e. it allows the user to detect subtle differences between the datasets. ${ }^{31}$ Following the application of PCA, a series of scores and loadings are produced. The first score, i.e. PC1, describes the maximum spectral variance in the dataset. This is followed by the second contains score (PC2) which describes the second most spectral variance in the data and so on. Thus, as the scores increase, their order of importance decreases. ${ }^{27,31}$ Distinct groupings are observed on the scores plots which can then be used to reflect the differences between samples. Typically, the scores plotted should be those that describe the maximum variance, i.e. PC1 and PC2 ${ }^{32}$ In this instance PCA was used to determine if the handheld spectrometer was detecting the SERS nanotags obscured by the plastic barriers. We have previously shown that PCA is a useful tool for this means. ${ }^{13}$ Spectra were truncated and scaled, before application of the first order derivative coupled with Savitzky-Golay smoothing.

Figure 2 shows the scores plot for the tracking of dye 823 nanotags through $20 \mathrm{~mm}$ of clear PET (a) and through $9 \mathrm{~mm}$ of blue PP (b). On each of the scores plots, the pink cluster refers to the plastic reference spectra (e.g. $20 \mathrm{~mm}$ thick plastic) and the black cluster refers to dye 823 nanotags obscured by plastic of the same thickness, i.e. 20 $\mathrm{mm}$. Convincing separation across PC1 is observed, thus indicating that the instrument is capable of detecting dye 823 SERRS nanotags through $20 \mathrm{~mm}$ of clear PET and $9 \mathrm{~mm}$ of blue PP using conventional Raman. It is worth noting that as the thickness of plastic increases the score for PC1, which indicates the maximum variance, decreases. This is expected since it demonstrates a decline in variability between reference plastic spectra (e.g. $20 \mathrm{~mm}$ thick PET) and spectra obtained of the nanotags obscured by plastic of the same thickness, thus offering further validation 
that it becomes harder for the instrument to detect SERS nanotags as the thickness of plastic increases.
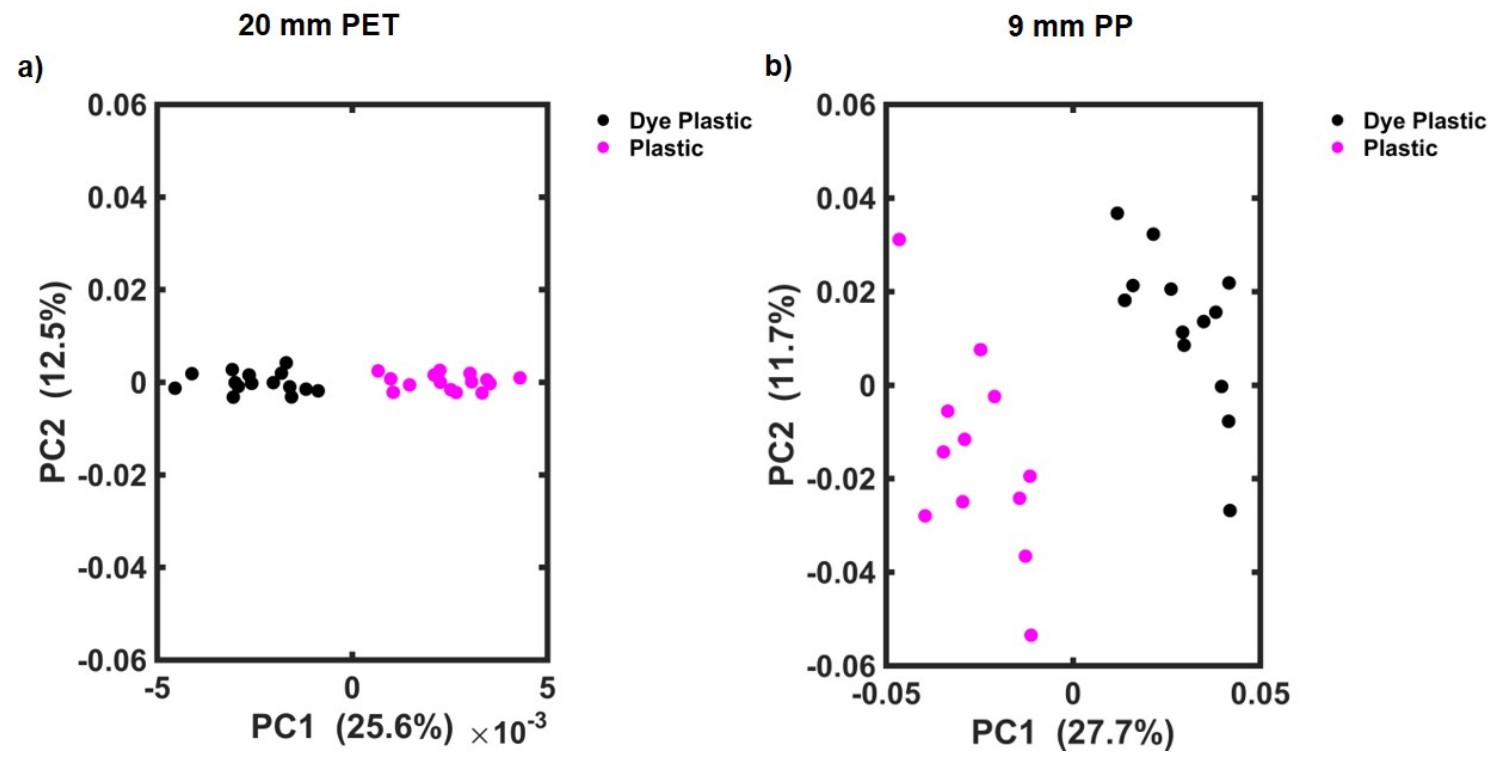

Figure 2 - PCA scores plots of the tracking of dye823 through $20 \mathrm{~mm}$ of PET and $9 \mathrm{~mm}$ of PP using a handheld conventional Raman instrument. In both instances, the pink clusters refer to plastic reference spectra at a given thickness (e.g. $20 \mathrm{~mm}$ ), and the black cluster refers to a solution of dye 823 nanotags obscured by the same thickness of plastic (e.g. $20 \mathrm{~mm}$ ). In both instances (PET and PP), clear separation is seen in the score plots indicating that the instrument is capable of detecting a solution of dye 823 nanotags through $20 \mathrm{~mm}$ of PET and $9 \mathrm{~mm}$ of blue PP.

At thicknesses beyond this, i.e. $21 \mathrm{~mm}$ clear PET and $10 \mathrm{~mm}$ of blue PP, separation is no longer seen in the scores plot (supporting information, Figure S6), indicating that the Raman instrument is no longer capable of detecting the dye 823 SERRS nanotags through greater barrier thicknesses. Nonetheless, the depth penetration capabilities of this handheld Raman instrument to detect SERRS analytes using a conventional Raman configuration through large thicknesses, without the need for SORS, is presented. These results suggest that SORS techniques may not always be necessary for probing through barriers. In this instance, using conventional Raman spectroscopy, SERRS nanotags were detected through depths of $20 \mathrm{~mm}$ of PET. However, the likelihood that nanotags would need to be tracked through plastic with such a large thickness in a real world situation is slim. Thus, since the majority of plastic containers are only a few $\mathrm{mm}$ thick, ${ }^{15}$ the results presented here demonstrate the potential of handheld conventional Raman to probe through relevant thicknesses without the need for more complex and expensive optical configuration, such as SORS. In addition, it is clearly demonstrated that using multivariate analysis greatly improves the ability to acquire spectral information and probe through larger thicknesses compared to what can be detected solely by analysing the data by eye.

It is well established that by using a Raman reporter that has an electronic transition that corresponds to the excitation wavelength of the laser, superior enhancement in Raman signal is generated. ${ }^{33,34}$ As already stated, the handheld instrument used in this work has a laser wavelength of $785 \mathrm{~nm}$, thus dye 823 is considered to be in resonance with the laser line. Based on this, dye 676, dye 959, BPE and AZPY are considered to be off resonant reporter molecules. Therefore, taking the signal 
enhancing benefits of SERRS into account, the ability to detect non-resonant Raman reporters through large thicknesses of plastic was also investigated. In order to demonstrate the advantage of conventional Raman for detection of nanotags functionalised with different Raman reporters at depth, the maximum thickness of plastic through which nanotags functionalised with dye 676, dye 959, BPE and AZPY was determined. The maximum depth that these nanotags could be tracked through was assessed using the same set up used for the assessment of dye 823, i.e. only the choice of Raman reporter differed, and the final concentration of dye was kept constant (300 nM).

The largest thicknesses of clear PET and blue PP that each of the SERS nanotags were detected through using handheld conventional Raman combined with PCA analysis are shown in Table 1. Dye 676, which is absorbs at $676 \mathrm{~nm}$, was successfully detected through $19 \mathrm{~mm}$ and $7 \mathrm{~mm}$ of PET and PP respectively. This was followed by dye 959, which absorbs at $959 \mathrm{~nm}$, and was tracked through PET thicknesses of 17 $\mathrm{mm}$ and PP thicknesses of $5 \mathrm{~mm}$. Following this, the suitability of the two non-resonant small molecules for through barrier detection applications was also determined. BPE, which has previously been shown to give a good SERS response in the NIR, ${ }^{26}$ was detectable through $11 \mathrm{~mm}$ of PET and $2 \mathrm{~mm}$ of PP. This was followed by AZPY which was detected through only $3 \mathrm{~mm}$ of PET and $1 \mathrm{~mm}$ PP, and thus generated the weakest SERS response through the two barriers.

Table 1 - The maximum thickness of clear PET and blue PP that nanotag solutions of dye 676, dye 823 , dye 959, BPE or AZPY were detected through using PCA. Nanotag solutions were prepared by functionalising AuNPs with each of the five Raman reporter molecules, i.e. dye 676 nanotag solution contained AuNPs functionalised with dye 676. The dyes are named according to their absorption maximum, thus dye 823 is resonant at $823 \mathrm{~nm}$ and is in resonance with the laser wavelength at $785 \mathrm{~nm}$. Measurements were performed using 3 samples, 5 replicates, at a laser excitation wavelength of $785 \mathrm{~nm}$, average laser power $43 \mathrm{~mW}$, and 3-s integration time, five accumulations.

\begin{tabular}{|c|c|c|}
\hline $\begin{array}{c}\text { Raman } \\
\text { reporter } \\
\text { molecule } \\
(\mathbf{3 0 0} \mathbf{~ n M )}\end{array}$ & $\begin{array}{c}\text { Thickness of } \\
\text { clear PET that } \\
\text { each nanotag } \\
\text { was detected } \\
\text { through }(\mathbf{m m})\end{array}$ & $\begin{array}{c}\text { Thickness of } \\
\text { blue PP that } \\
\text { each nanotag } \\
\text { was detected } \\
\text { through } \\
\text { (mm) }\end{array}$ \\
\hline $\mathbf{6 7 6}$ & 19 & 7 \\
\hline $\mathbf{8 2 3}$ & 20 & 9 \\
\hline $\mathbf{9 5 9}$ & 17 & 5 \\
\hline BPE & 11 & 2 \\
\hline AZPY & 3 & 1 \\
\hline
\end{tabular}

The results presented in Table 1 not only demonstrate the advantage of using a resonant Raman reporter for enhanced levels of through barrier detection, but also the benefit of using chalcogenpyrylium-based dyes, over commercially available small molecules, for probing through deeper depths. Overall, superior levels of through barrier detection are achieved when chalcogenpyrylium-based dyes are used and this supports previous work which has explored the use of the same dyes as SERS nanotags at $1280 \mathrm{~nm} .{ }^{26}$ All three chalcogenpyrylium-based dyes out-perform the commercially available small molecules BPE and AZPY despite dye 676 and dye 959 being off resonant with the $785 \mathrm{~nm}$ excitation wavelength. The ability of dye 676 and 
dye 959 as well as the resonant dye 823 to be detected through large thicknesses of plastic is due to their structural properties in which high polarisability exists. As such, they exhibit large Raman cross sections and are therefore exceptional Raman scatters. Thus, by exploiting these properties to support through barrier detection applications, chalcogenpyrylium-based SERS nanotags were capable of being detected through larger thicknesses of plastic than commercially available reporters. This work demonstrated that in comparison to the commercially available nonresonant Raman reporters BPE and AZPY, chalcogenpyrylium-based dyes are much more effective Raman reporters at longer wavelengths. ${ }^{26}$ Both these Raman reports have excitation wavelengths that are close to the $785 \mathrm{~nm}$ excitation wavelength of the Raman instrument, with dye 823 being closest to resonance. It was observed that both dye 676 and dye 823 generate similar levels of through barrier detection, i.e. they can be detected through similar thicknesses of PET and PP. It is hypothesised that the smaller size of dye 676 compared to dye 823 (one sp² carbon versus three $\mathrm{sp}^{2}$ carbons in the aliphatic backbone), means that a greater number of dye molecules can achieve favourable steric arrangements when they interact with the gold surface. Thus, despite dye 676 being further from resonance with the laser excitation wavelength, similar levels of though barrier detection are achieved. Furthermore, it is also worth noting that it was not possible to apply a different point and shoot adaptor lens to the instrument which might afford a greater (or lesser) focal distance. If an adaptor with a longer focal distance was available, it is feasible that through barrier detection would be achieved through even greater thicknesses, particularly with regards to PET since its transparent nature will allow beam penetration to greater depths. Nonetheless, the work presented here demonstrates the significant potential of handheld Raman to see through large thicknesses of plastic barriers.

Based on these results, it was anticipated that the most suitable Raman reporter for detection of nanotags in through tissue applications would also be dye 823. To confirm this, solutions of each of the five nanotags were held in Suprasil quartz micro cuvette, with a path length $1 \mathrm{~mm}$ and a chamber volume $350 \mu \mathrm{L}$. The experimental set up is shown in the supporting information, Figure S4. In keeping with the experiments involving plastic, the final concentration of each of the five Raman reporter molecules was kept at $300 \mathrm{nM}$. A tissue section (porcine) was placed in front of the nanotags in the cuvette and the point and shoot adapter of the instrument was brought into contact with the tissue. The height of the most intense peak in the spectrum of each of the five Raman reporters was calculated, as well as the relative percentage peak intensity (Figure 3). Since dye 823 generated the strongest intensity, it is assigned an intensity value of $100 \%$. The relative peak intensity refers to the peak intensity of nanotags containing dye 676 , dye 959 , BPE or AZPY obscured by $5 \mathrm{~mm}$ of tissue, relative to the peak intensity seen using dye 823 . The remaining four peaks are expressed as a percentage relative to that value. As already stated, the handheld Raman instrument has an excitation wavelength of $785 \mathrm{~nm}$, thus the results presented in Figure 3 demonstrate that there is a substantial increase in signal with the use of a resonant Raman reporter for probing through tissue barriers, with dye 823 and dye 676 giving the largest signal. 


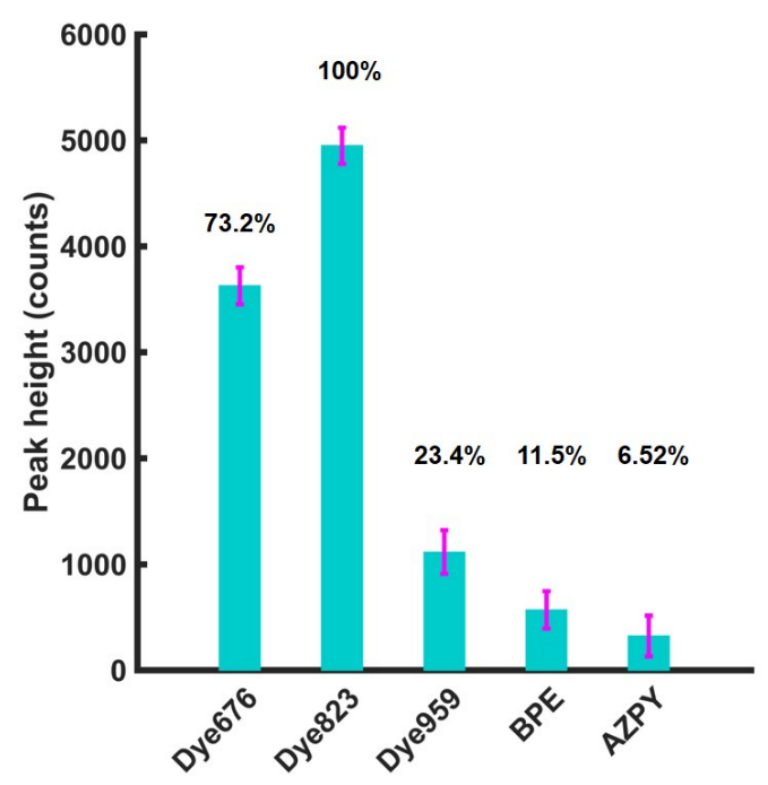

Figure 3 - Bar chart showing average peak intensities of dye 676, dye 823, dye 959, BPE and AZPY at 1601, $1596,1581,1609$ and $1162 \mathrm{~cm}^{-1}$ respectively, as well as the relative percentage peak intensity relative to the most intense signal from dye 823, through $5 \mathrm{~mm}$ of tissue. Nanotag solutions were held in a cuvette and the cuvette was placed behind tissue samples. Spectra were collected using a handheld CBEx instrument with $785 \mathrm{~nm}$ laser excitation. Peak intensities were obtained by scanning 3 replicate samples, 5 times (5 second integration time). The average peak intensity for each of the 5 dyes is shown and error bars represent one standard deviation.

Having established that the resonant dye 823 nanotags gave the most intense SERS response, the maximum thickness of tissue that dye 823 nanotags could be detected through was then investigated. To establish this, the nanotags were held in a cuvette and obscured by varying thicknesses of porcine tissue, Figure 4 shows the data obtained through $10 \mathrm{~mm}$ of porcine tissue. The characteristic dye peak at $1596 \mathrm{~cm}^{-1}$ is clearly visible by eye and thus it can be confidently established that the handheld Raman instrument is capable of detecting the nanotags through $10 \mathrm{~mm}$ of tissue. We have previously reported the ability to track the same SERRS nanotags through depths of $25 \mathrm{~mm}$ of tissue using a similar experimental set-up using SORS rather than conventional Raman. ${ }^{22}$ Although the depth penetration achieved here is less than that achieved using the SESORRS technique, the results presented here are impressive for conventional Raman, particularly since the focal distance of the handheld conventional Raman instrument is fixed. It is again anticipated that if a point and shoot adaptor with a longer working distance was available, dye 823 nanotags could potentially be detected through even greater thicknesses of tissue. Furthermore, these results show the significant potential of handheld Raman for the probing of SERRS nanotags at clinically relevant depths in vivo. 


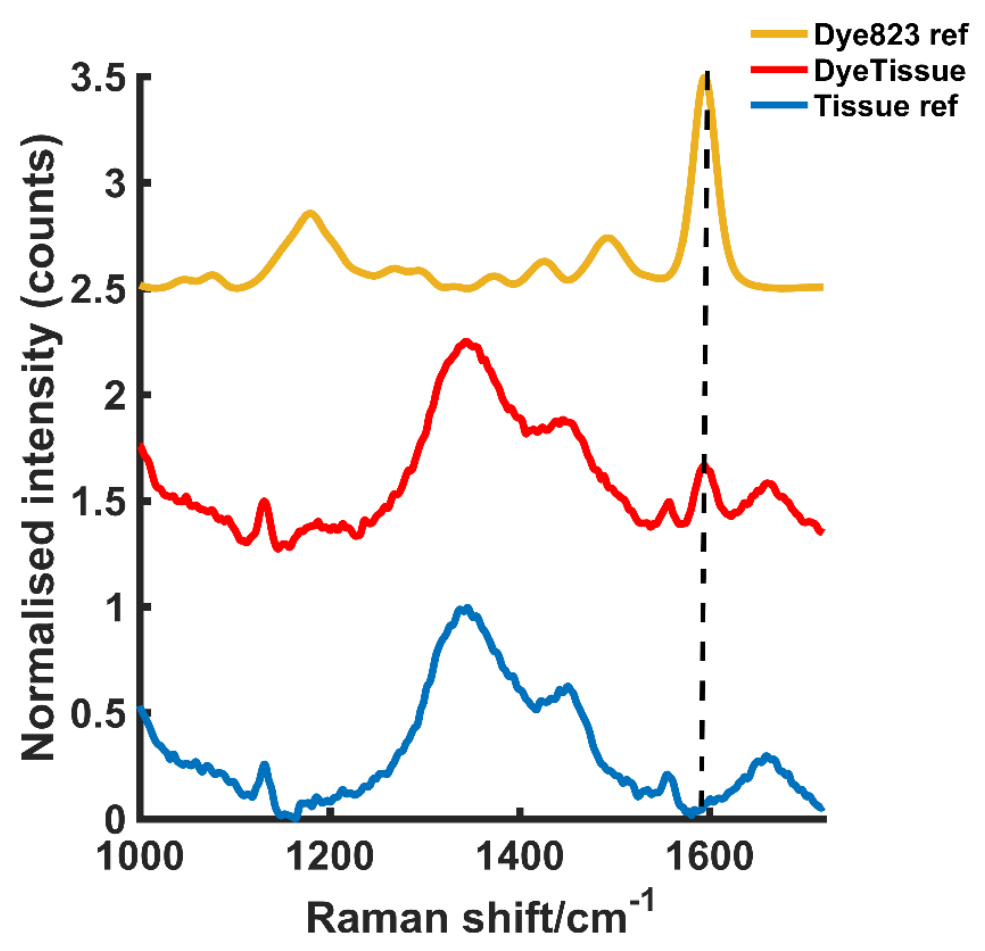

Figure 4 - The tracking of dye 823 nanotag solution through $10 \mathrm{~mm}$ of tissue. The tissue and dye 823 reference spectra are shown at the bottom and top respectively. The middle spectrum represents the Raman signal collected from the nanotags obscured by $10 \mathrm{~mm}$ of tissue. The peak at $1596 \mathrm{~cm}^{-1}$ is easily detectable by eye. Spectra were collected using a handheld CBEx instrument with $785 \mathrm{~nm}$ laser excitation, $5 \mathrm{~s}$ integration. Peak intensities were obtained by scanning 3 replicate samples, 5 times.

However, detecting the nanotag solution in the confined environment of a cuvette, where the NPs are not dispersed over an area or free to move around does not truly represent a biological system. In this instance the nanotags would be subject to dispersion within the matrix as well as intracellular processes which may limit their uptake into cells, thus ultimately reducing the number of nanoparticles and therefore SERRS signal, at the point of measurement. Whilst this still mimics the potential to use SERRS to track nanotags in tissue, for example drug release systems where the NPs are embedded in a diffusion system such as a reservoir or matrix device, this is less representative of a system in which the nanotag solution is administered intravenously. Thus, to further mimic the potential to use handheld Raman to detect nanotags in vivo, we used multicellular tumour spheroids (MTS) as ex vivo breast cancer tumour models. Unlike two dimensional (2D) cell cultures, MTS establish characteristic concentration gradients of oxygen, nutrients and metabolites and thus can be used as an ex vivo tumour model as they more closely resemble the 3D in vivo environment. ${ }^{35,36}$ Due to the enhanced permeability and retention (EPR) effect, there is potential for NPs to accumulate preferentially in tumours, therefore NPs have potential for use as drug delivery platforms ${ }^{37}$ and to support imaging applications. ${ }^{38}$ MTS can provide a model for NP accumulation in tumours in vivo without the need for more complex ethical approval and long term experiments associated with such studies.

MTS were used as an ex vivo breast cancer model to demonstrate the ability to detect SERRS nanotags at depth using handheld Raman. MCF7 human breast cancer cells were incubated overnight with dye 823 nanotags. MTS were then grown using a hanging drop technique by pipetting $20 \mathrm{~mL}$ drops of MCF7 human breast cancer cell 
suspension onto the lid of a Petri dish. They were grown over a period of 7 days at 37 $\mathrm{C}$ and $5 \% \mathrm{CO}_{2}$ in a humified incubator to a size $<1 \mathrm{~mm}$. No reduction in growth was observed. It is therefore reasonable to assume that the dyes did not cause cell death since cells need to be alive in order to divide and replicate to form MTS models.

Roughly 10 MTS were transferred to a single section of tissue. Following this, another layer of porcine tissue with a $5 \mathrm{~mm}$ thickness was then placed on top of the MTS imbedded tissue. The two-layer tissue sample was then transferred to an $x-y$ translational stage and the handheld Raman instrument was positioned perpendicular to the tissue section to enable the Raman mapping of the dye 823 SERRS nanotags taken up into the ex vivo breast cancer tumour models through $5 \mathrm{~mm}$ off tissue. The experimental set up is described in Figure S5, supporting information. The tissue samples were moved in steps of $1 \mathrm{~mm}$ to create an image of $10 \times 10$ pixels. It is important to note that the z-direction remained fixed and the point and shoot adaptor, with a working distance of $5 \mathrm{~mm}$ was used to probe through the tissue barrier. A false colour 2D SERRS heat map was then constructed, Figure $5 a$. This corresponds to the uptake of dye 823 nanotags into MTS. The map demonstrates clear discrimination between areas where the MTS models were present (i.e. placed onto the tissue section), and areas where they were not. This is confirmed in the spectrum collected at the point of maximum intensity (Figure $5 \mathrm{~b}$ ) where the characteristic dye 823 peak at $1596 \mathrm{~cm}^{-1}$ is observed. In areas where the MTS containing the SERRS nanotags were not present, no spectral contribution from the dye is seen. In this instance, the observed spectrum corresponds to that of the tissue only. Therefore, since in this model system, the precise location of the spheroids in the tissue is known, control spectra can be generated on the same image but away from the MTS deposition point. It should also be noted that the SERRS-active NPs were contained within the spheroids themselves and therefore their location was known prior to imaging. In comparison to the experimental set up involving a bulk set up, i.e. nanotags held in a vial or a cuvette, the number of NPs present in each MTS was significantly less. Therefore, the results presented here demonstrate the on/off detection of SERRS nanotags in areas where they are present in MTS and where the MTS models are not present by monitoring the peak intensity at $1596 \mathrm{~cm}^{-1}$ through thicknesses of $5 \mathrm{~mm}$. These results are particularly impressive since a handheld Raman spectrometer has been utilised to detect the SERRS signal through diffusely scattering turbid media. A previous report has demonstrated the potential to detect the same SERRS nanotags take up into MTS through depths of $15 \mathrm{~mm}$ using SESORRS, ${ }^{22}$ however it is expected that advancements in instrumentation will increase the potential of handheld Raman to probe through clinically relevant depths.

The work presented here has explored the use of large gold AuNPs as SERS substrates for through barrier detection. Spherical AuNPs were chosen based on their stability and high scattering cross sections however it is well established that several other metallic nanostructures can be synthesised with tunable plasmons in the NIR region. ${ }^{11}$ However, we have previously compared the use of $100 \mathrm{~nm}$ gold nanoparticles and hollow gold nanoparticles as SERS substrates at longer wavelengths $(1550 \mathrm{~nm})$, in combination with the same chalcogenpyrylium used in this work. ${ }^{1}$ The results showed that $100 \mathrm{~nm}$ AuNPs outperformed hollow gold nanoparticles which had a tunable LSPR in the NIR region. 
a)

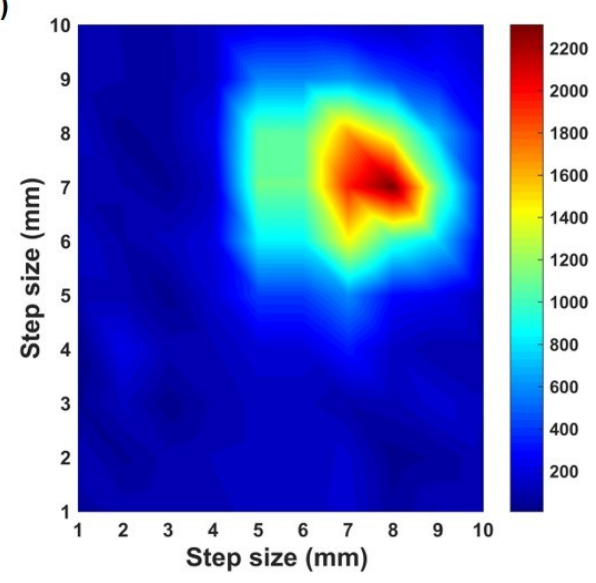

b)

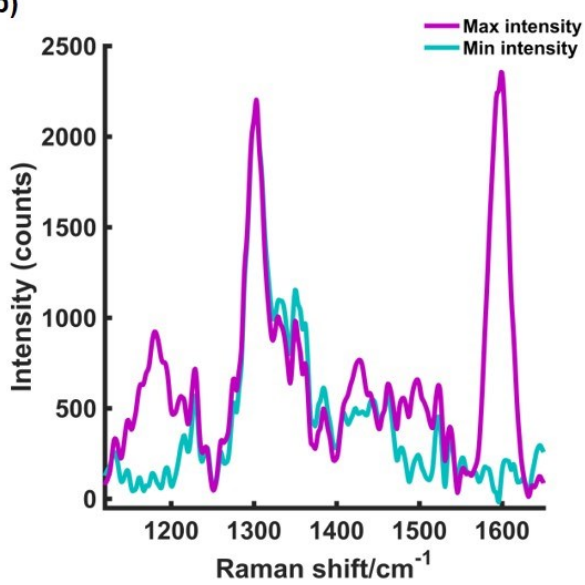

Figure 5 - a) A false colour xy-2D heat SERRS map of MTS containing dye 823 through $5 \mathrm{~mm}$ of tissue. The map was constructed using the peak intensity at $1596 \mathrm{~cm}^{-1}$. Measurements were carried out using an $x y$ translational stage and moving it in step sizes of $1 \mathrm{~mm}$ to create an image of $10 \times 10$ pixels. Spectra were truncated, baselined and smoothed prior to processing. A combination surface/contour false colour was used to generate a 2D heat map and show the tracking of the MTS through $5 \mathrm{~mm}$ of tissue. Clear discrimination is seen between spectra collected at the point of maximum intensity where the nanotags containing MTS models were spotted, and that collected where the MTS were not present. (b) The corresponding maximum and minimum collected through $5 \mathrm{~mm}$ of tissue. offset spectra. All measurements were carried out using a $5 \mathrm{~s}$ integration time, $785 \mathrm{~nm}$ laser excitation wavelength.

\section{Conclusions}

Through utilising the powerful Raman scattering properties of chalcogenpyrylium reporters for SERRS applications, the ability to probe through large thicknesses of plastic and tissue is presented. To the best of our knowledge, this is the first assessment of the maximum thickness that this handheld spectrometer can detect SERRS nanotags through. Furthermore, it is the first assessment of the benefit of using a resonant Raman reporter to probe through the plastic and tissue barriers using SERRS and in addition, the first report of the detection of 3D tumour models through tissue barriers using handheld conventional Raman. In combination with PCA, the ability to detect SERRS nanotags through up to $20 \mathrm{~mm}$ of plastic is demonstrated, alongside the tracking of SERRS nanotags through up to $10 \mathrm{~mm}$ of tissue using handheld Raman.

Previous work involving the detection of SERRS nanotags through tissue has involved the use of SORS or transmission Raman therefore the results used here are extremely promising as conventional Raman was utilised for the detection of nanotags through $5 \mathrm{~mm}$ of tissue. This work demonstrates the potential of conventional Raman to probe through large thicknesses using specifically designed SERRS nanotags tuned to match the excitation wavelength. In addition, it demonstrates that the use of SORS instrumentation to detect SERS nanotags may not always be necessary when probing through barriers, particularly plastic. Conventional Raman instruments are more widely available in spectroscopy laboratories than SORS instrumentation, therefore we demonstrate that if SORS instrumentation is not accessible, conventional Raman instrumentation may be just as useful in through barrier detection applications, particularly if this is combined with the use of a resonant Raman reporter molecule. In addition, the instrument used here was handheld and relatively low cost compared to handheld SORS and its small size and portability lends itself well to clinical 
applications. Therefore we envisage that future development of handheld Raman instrumentation will go hand in hand with increased clinical acceptance of Raman spectroscopy by regulatory bodies worldwide.

\section{Acknowledgements}

The authors thank Prof Val Brunton and Anastasia Kapara at the IGMM, University of Edinburgh, for providing the cell line.

\section{Conflict of Interest}

The authors declare no conflict of interest

\section{Funding}

This work was supported by Dstl and the Engineering and Physical Sciences Research Council [grant numbers EP/J500550/1 and EP/M506643/1, KF and FN and EP/L014165/1, SM, DG and KF] and by the National Science Foundation [grant number CHE-1566142, KP and MRD]. Research data associated with this paper will become available through the following link: http://dx.doi.org/10.15129/563a0bd91231-4e4e-b6dc-10a989dd5df2

\section{References}

1 H. Kearns, M. A. Bedics, N. C. Shand, K. Faulds, M. R. Detty and D. Graham, Analyst, 2016, 141, 5062-5065.

2 G. McNay, D. Eustace, W. E. Smith, K. Faulds and D. Graham, Appl. Spectrosc., 2011, 65, 825-837.

3 H. Kearns, R. Goodacre, L. E. Jamieson, D. Graham and K. Faulds, Anal. Chem., 2017, 89, 12666-12673.

4 D. Cowcher, Y. Xu and R. Goodacre, Anal. Chem., 2013.

$5 \quad$ X. Qian, X.-H. Peng, D. O. Ansari, Q. Yin-Goen, G. Z. Chen, D. M. Shin, L. Yang, A. N. Young, M. D. Wang and S. Nie, Nat. Biotechnol., 2008, 26, 83-90.

6 T. Demeritte, R. Kanchanapally, Z. Fan, A. K. Singh, D. Senapati, M. Dubey, E. Zakar and P. C. Ray, Analyst, 2012, 137, 5041.

7 J. M. Sylvia, J. A. Janni, J. D. Klein and K. M. Spencer, Anal. Chem., 2000, 72, 5834-5840.

8 D. L. Ellis, H. Muhamadali, S. A. Haughey, C. . Elliot and R. Goodacre, Anal. methods, 2015, 7, 9401-9414.

9 A. Oseledchyk, C. Andreou, M. A. Wall and M. F. Kircher, ACS Nano, 2017, $11,1488$.

10 C. Decristoforo, B. Faintuch-Linkowski, A. Rey, E. von Guggenberg, M. Rupprich, I. Hernandez-Gonzales, T. Rodrigo and R. Haubner, Nucl. Med. 
Biol., 2006, 33, 945-52.

11 S. Laing, L. E. Jamieson, K. Faulds and D. Graham, Nat. Rev. Chem., 2017, 1, 0060 .

12 E. Smith and G. Dent, Modern Raman Spectroscopy: A Practical Approach, Wiley, 2005.

13 F. Nicolson, L. E. Jamieson, S. Mabbott, N. C. Shand, D. Graham and K. Faulds, J. Raman Spectrosc., 2017, 48, 1828-1838.

14 N. C. Shand, SPIE Eur. Secur. Def., 2008, 7119, 71190J-71190J-12.

15 M. Bloomfield, P. W. Loeffen and P. Matousek, Proc. SPIE, 2010, 7838, 783808-1-783808-15.

16 K. Buckley, C. G. Atkins, D. Chen, H. G. Schulze, D. V Devine, M. W. Blades and R. F. B. Turner, Analyst, 2016, 141, 1678.

17 N. Stone, R. Baker, K. Rogers, A. W. Parker and P. Matousek, Analyst, 2007, 132, 899-905.

18 M. V Schulmerich, K. a Dooley, M. D. Morris, T. M. Vanasse and S. a Goldstein, J. Biomed. Opt., 2006, 11, 060502.

19 P. Matousek, E. Draper, A. Goodship, I. Clark, K. Ronayne and A. W. Parker, Appl. Spectrosc., 2006, 60, 758-763.

20 N. Stone, K. Faulds, D. Graham and P. Matousek, Anal. Chem., 2010, 82, 3969-3973.

21 N. Stone, M. Kerssens, G. R. Lloyd, K. Faulds, D. Graham and P. Matousek, Chem. Sci., 2011, 2, 776-780.

22 F. Nicolson, L. E. Jamieson, S. Mabbott, K. Plakas, N. Shand, M. Detty, D. Graham and K. Faulds, Chem. Sci., 2018, 9, 3788-3792.

23 F. Nicolson, L. E. Jamieosn, S. Mabbott, K. Plakas, N. C. Shand, D. Graham and K. Faulds, Chem. Commun., 2018, 54, 8530-8533.

24 D. Kurouski and R. Van Duyne, Anal. Chem., 2015, 87, 2901-2906.

25 S. Zaleski, K. A. Clark, M. M. Smith, J. Y. Eilert, M. Doty and R. P. Van Duyne, Anal. Chem., 2017, 89, 2497-2504.

26 M. A. Bedics, H. Kearns, J. M. Cox, S. Mabbott, F. Ali, N. C. Shand, K. Faulds, J. B. Benedict, D. Graham and M. R. Detty, Chem. Sci., 2015, 6, 2302-2306.

27 H. J. Butler, L. Ashton, B. Bird, G. Cinque, K. Curtis, J. Dorney, K. Esmondewhite, N. J. Fullwood, B. Gardner, P. L. Martin-Hirsch, M. J. Walsh, M. R. Mcainsh, N. Stone and F. L. Martin, Nat. Protoc., 2016, 11, 664-687.

28 S. Moreton, K. Faulds, N. Shand, M. Bedics, M. Detty and D. Graham, Nanoscale, 2015, 6075-6082.

29 D. I. Ellis, R. Eccles, Y. Xu, J. Griffen, H. Muhamadali, P. Matousek, I. Goodall and R. Goodacre, Sci. Rep., 2017, 7, 12082. 
P. Matousek, TrAC Trends Anal. Chem., 2018, 103, 209-214.

31 R. Goodacre, D. Graham and K. Faulds, TrAC - Trends Anal. Chem., 2018, 102, 359-368.

32 M. Richardson and A. K. Smilde, Anal. methods, 2009, 2014, 2812-2831.

33 B. Sharma, R. R. Frontiera, A.-I. Henry, E. Ringe and R. P. Van Duyne, Mater. Today, 2012, 15, 16-25.

34 S. Harmsen, M. A. Bedics, M. A. Wall, R. Huang, M. R. Detty and M. F. Kircher, Nat. Commun., 2015, 6, 6570.

35 L. E. Jamieson, D. J. Harrison and C. J. Campbell, Analyst, 2015, 140, 39103920.

36 L. E. Jamieson, V. L. Camus, P. O. Bagnaninchi, K. M. Fisher, G. D. Stewart, W. H. Nailon, D. B. McLaren, D. J. Harrison and C. J. Campbell, Nanoscale, 2016, 8, 16710-16718.

37 E. Blanco, H. Shen and M. Ferrari, Nat. Biotechnol., 2015, 33, 941-951.

38 A. B. Chinen, C. M. Guan, J. R. Ferrer, S. N. Barnaby, T. J. Merkel and C. A. Mirkin, Chem Rev, 2015, 115, 10530-10574. 